\title{
Low field NMR Time Domain (TD) Characterization of PUFA-rich Linseed and Fish Oil Emulsions During Thermal Air Oxidation
}

\author{
Maysa Resende ${ }^{1}$, Charles Linder ${ }^{1}$, and Zeev Wiesman ${ }^{1}$ \\ ${ }^{1} \mathrm{BGU}$
}

September 16, 2020

\begin{abstract}
Linseeds contains high levels of PUFA $\alpha$-linolenic acid, naturally protected against thermal oxidation by their encapsulation within LS oil bodies by multiple components including antioxidant proteins and mucilage emulsifying agents. By LS grinding, adding of water, adjusting pH, and sonication LS oil bodies emulsions (LSE) can be formed which can also encapsulate externally added PUFAs, to minimize their thermal oxidation, as it does for the intrinsic ALA PUFAs. Fish oil encapsulation into this LSE platform (LSFE) offers the possibility of a nutritive delivery system of the biologically essential PUFA fish oil's, protected from oxidation, which to date is difficult to achieve. In this study structural and chemical properties LF $1 \mathrm{H}$ NMR T1-T2 characterization of LSE and LSFE was used to analyze their stability and changes, under thermal oxidizing conditions. Peak changes in these LF $1 \mathrm{H}-\mathrm{NMR}$ spectra were correlated with the stability of chemical and physical variables during thermal (55oC for $96 \mathrm{hrs}$ ) oxidation. The present study demonstrates the capability of $1 \mathrm{H}$ LF-NMR relaxation sensor to monitor the time domain fingerprints of chemical and structural changes of LSE and with co-encapsulated fish oil (LSFE) under thermal autoxidation conditions. The results of the LF-1H NMR analysis are further supported and correlated with conventional peroxide value tests, self-diffusion, droplets size distribution, zeta potential estimation of surface stability under thermal oxidation conditions. The results of this study demonstrate the efficacy of LSE to minimize linseed and encapsulated fish oil PUFA oxidation.
\end{abstract}

\section{Introduction}

The present study is focused on characterization of linseed oil body emulsions for prevention of polyunsaturated fatty acid (PUFA) oxidation in food products and supplements. Its importance is due to healthy nutrition requires consumption of the omega-3 polyunsaturated fatty acids (PUFA) $\alpha$-linolenic acid (ALA), eicosapentaenoic acid (EPA) and docosahexaenoic acid (DHA) that are key nutrients involved in normal growth and development of various human tissues (Zarate et al., 2017; Yesiltas et al., 2019). They, however are not synthesized within the body but can be obtained from plant and marine sources, such as linseeds that have been widely used since ancient times for different practical and valuable applications including healthy foods. Linseeds health feature is their high oil content of PUFAs, including omega-3 (ALA) and omega- 6 fatty acids. Since the consumption of omega-3 fatty acids has diverse beneficial human health effects (Abozid and Ayimba, 2014) linseed oil is used in pharmaceutical products, cosmetics and food supplements. Other important linseed components: the fiber fractions of the seeds contain the antioxidant lignan that may contribute to the prevention of certain cancers (Thompson and Cunnane, 2003). They also contain mucilages with anti-inflammatory effects on the intestine and seem to decrease cholesterol and glucose levels in the blood (Thakur et al., 2009). Linseed proteins, rich in arginine, are thought to have antioxidant and antiatherogenic properties.

Linseed oils due to its high PUFA levels are well known to undergo oxidation polymerization, conferring drying properties, which are used in diverse technical applications, including paints, mastic formulations and wood treatment (Jhala and Hall, 2010; Humar and Lesar, 2013). Paradoxically, PUFAs though needed 
for health, also undergo facile oxidation during storage and digestion into toxic oxidative products (Yehuda et al., 1999). Therefore, methods to overcome oxidation such as protective encapsulation and the use of antioxidants is extensively studied (Jackowski et al., 2015 and Jacobsen, 2015). One delivery approach is based on PUFA-rich oils encapsulated in gelatin capsules, but for salmon fish oil, because of its high EPA and DHA concentration it is still sensitive to shelf life oxidation. An alternative relatively novel approach to PUFA encapsulation is based on natural seed oil bodies (OB), that can spontaneously, under external force, organize in a water matrix to form emulsions (Goyal et al., 2015). Linseed is an excellent source of such oil body emulsion products (Fabre et al 2015).

Dispersion of aqueous extracts of intact seed oil bodies (OBs) in water is a novel way of producing natural and oxidatively stable food emulsions with minimal use of synthetic antioxidants and emulsifiers, as there is growing interest in natural food emulsions containing unsaturated oil (Shen et al., 2012). Within linseeds, the oil is stored in OB, which protects the PUFA triacylglycerides (TAG) (97.6\% of the mass of the oil body) by their encapsulation with a membrane composed of phospholipids $(0.9 \%)$ and proteins $(1.34 \%)$ (Tzen and Huang, 1992). The oil bodies are stabilized and protected against coalescence by the proteins present in the membrane; they can be directly incorporated into formulations and this process is facilitated in some cases by the emulsifying compounds present in the seed. Oil bodies and tensioactive proteins are generally extracted in water from the seed. The exceptional physical stability of Linseed OBs has been attributed to unique amphipathic proteins called oleosins that occur within the oil body surface (Huang, 1994). They are thought to stabilize OBs by steric hindrance and electronegative repulsion (Jolivet et al., 2009). It has been suggested that the entire surface of the OB is covered by oleosin such that the compressed OBs rarely coalesce or aggregate in the cells of a mature seed (Tzen and Huang, 1992). The storage of TAGs within OBs not only prevents the TAG droplets from aggregation and coalescence but also appears to protect them from lipid oxidation. OBs protect lipid reserves against oxidation and hydrolysis until seed germination and seedling establishment (Purkrtova et al., 2008). Oils of linseed, sunflower and echium contain highly levels of PUFA that very susceptible to oxidative deterioration resulting in a rapid decrease in palatability, nutritional quality and shelf-life of foods into which they are incorporated. Intact OBs offer a novel and effective route to the preparation of oxidatively stable food emulsions from such unstable PUFA oils without necessarily using synthetic antioxidants for stabilization. Current international dietary guidelines advocate replacement of saturated fats in food with unsaturated fats which heightens the need to develop more efficient and improved methods for stabilization of unsaturated oils incorporated in to emulsion-based foods.

In nuts and seeds, lipids are stored in oil bodies called oleosomes that store energy in the form of TAGs for use during germination and to protect the lipids against physical and chemical stresses in the seeds (Nikiforidis, 2019). Nature has evolved oleosomes as efficient mechanism against lipid oxidation via an encapsulation by relatively complex phospholipid/protein membranes, that are the only membranes that consist only of a monolayer of phospholipids anchored with proteins (Karefyllakis et al., 2019). The phospholipid layer has a thickness of about $0.9 \mathrm{~nm}$ and composes about $2 \mathrm{wt} \%$ of the total mass of the seed oleosomes (Millichip et al., 1996). Within and on this layer are proteins (e.g., caleosins, and steroleosins (Nikiforidis, 2019; Karefyllakis et al., 2019; Wahlroos et al., 2015). The proteins N-terminal (N) and C-terminal (C) domains associate with the phospholipid polar heads.

The present study characterizes linseed ALA fatty acid OBs, as an encapsulating carrier of relatively high levels of EPA and DHA from fish oil that are highly susceptible to oxidation. The OB's phospholipids and amphiphilic proteins and peptides of oleosin from linseed are well known to spontaneously form an encapsulating shell around the oil emulsion particles, with antioxidant characteristics (Fisk et al., 2008). These linseed's emulsion of linseed oil and the amphiphilic components of the linseed (phospholipids and proteins) that stabilize the emulsion formed by sonication of the seeds within water will be characterized by the methodologies described below and will be correlated to its antioxidant properties for the encapsulated PUFA's. In addition, the different components of linseeds can contribute to the emulsion's lipophilic antioxidants/vitamins, and arrange according to interfacial forces such as tocopherol and lipophilic phenols and polyphenols within the oil phase, and within the water the phase are water soluble antioxidants/vitamins such as hydrophilic polyphenols and ascorbic acid (Shen et al., 2012). 
Numerous chemical and physical analytical methods have been developed to asses lipid oxidation such as conjugated diene values, peroxide values (PV), alcohols, epoxides, p-anisidine assay, HBR titration, iodometric titration, xynol orange, total polar components (TPC) by high performance liquid chromatography (HPLC), fatty acid composition determined by gas chromatography-mass spectrometry (GC-MS), fourier transformation infrared spectroscopy (FTIR), volatile product determination by gas chromatography, dimer/polymers by size exclusion chromatography (SEC), and electron spin resonance (ESR) (Jacobsen, 2015; Hwang et al., 2017; Velasco et al., 2005). There is however a lack of consistency in many of the results, because most of these analytical methods are designed to detect one type of oxidation product while lipid oxidation is a very complicated process producing numerous products at different times of oxidation. Hence, as suggested by Hwang et al. (2017), the development of methods that combine the concomitant detection of different types of oxidation products is necessary for the consistent assessment of lipid oxidation. In this respect, as described below, LF-1H-NMR spectroscopy technology has a significant potential in elucidating molecular structures of oxidation products from lipids and in revealing the mechanisms of lipid oxidation.

Methods using high field ${ }^{1} \mathrm{H}$ NMR relaxation were found by Sun et al. (2011) and Bakota et al. (2012) to correlate well the various parameters associated with lipid oxidation (e.g., free fatty acid; polar materials in heated oils; solid fat content). Hwang et al. (2017) proposed that "there are molecular structure and composition changes in oil during oil oxidation and degradation process affecting the chemical environment surrounding the protons. Thus, the proton mobility affecting the NMR energy relaxation time values changes as oil degrades". High field ${ }^{1} \mathrm{H}$ NMR was also used to analyze aldehydes produced in various heated oils (Guillen and Uriarte, 2009). These researchers reported on the ability to analyze a list of aldehyde products in linseed oil heated at $190{ }^{\circ} \mathrm{C}$ for $20 \mathrm{~h}$, and also determined acyl groups' iodine value and polar compounds. Merkx et al. 2018 reported a broad band selective ${ }^{1} \mathrm{H}$ NMR method for determination of both hydroperoxides and aldehydes in oxidized oils. Furthermore, based on electron spin resonance (ESR) system, combined with a free radical standard and trapping agents (TEMPO and PBN) was released for determination of peroxides in the early fast initiation phase of oil oxidation (Velasco et al., 2005). Blumich (2016) developed compact low field ${ }^{1} \mathrm{H}$ LFNMR systems and Gouilleux et al. (2016) developed an automate LF-NMR system. However, one of the remaining problems of ${ }^{1} \mathrm{H}$ LF $-\mathrm{NMR}$ and especially $2 \mathrm{D}$ T1-T2 systems is the relatively long experimental and data processing time required to finalize the results. Therefore, these systems were not suitable for high throughput applications such as real-time reaction monitoring or rapid screening of oil oxidation (Hwang et al., 2017).

In the present work, ${ }^{1} \mathrm{H}$ LF-NMR recently developed data processing was used to characterize both the chemical and physical/morphological structures directly on a single graph (Weisman et al., 2018, Resende et al 2019a,b). Our preliminary studies have shown how the ${ }^{1} \mathrm{H}$ LF-NMR energy relaxation time technology with L1/L2 norm regularization parameters can characterize and monitor PUFA rich oil oxidation, by generating 2D chemical and morphological spectra (Campisi et al. 2018, 2019; Resende et al., 2019a,b, 2020). In effect by using our recently developed primal-dual interior method for the convex objectives (PDCO) optimization solver for computational processing of the energy relaxation time signals $\mathrm{T}_{1}$ (spin-lattice) and $\mathrm{T}_{2}$ (spinspin), 2D spectra of the morphological and chemical arrangements could be reconstructed. The LF-NMR signal reconstruction into $2 \mathrm{D} \mathrm{T}_{1}$ vs. $\mathrm{T}_{2}$ graphs effectively characterizes the chemical and morphological domains of complex materials (Resende et., al 2019a) such as the 2D spectra generated for butter, rapeseed oil, soybean oil, and linseed oil. In order to correlate the chemical and morphology arrangements of these emulsified aggregate structures we used our previous work on TD NMR energy relaxation time analysis that can monitor changes in highly complex systems. These studies also showed how the different degrees of unsaturation of fatty-acid oils affects their chemical and morphological domains and influences their oxidative susceptibility (Resende et al., 2019a).

The aim of the present study is to demonstrate the capability of ${ }^{1} \mathrm{H}$ LF-NMR energy relaxation time sensor to monitor the TD fingerprints of chemical and structural changes of linseed oil bodies emulsion (LSE) and linseed emulsion enriched with fish oil (LSFE) in thermal autoxidation conditions and to test the efficacy of LSE as an autoxidation protected fish oil PUFA-rich delivery system. Specific objectives include characterization of structural and chemical changes and stability using TD peak assignment, under thermal oxidizing 
conditions $\left(55^{\circ} \mathrm{C}\right.$ for $\left.94 \mathrm{hrs}\right)$ using ${ }^{1} \mathrm{H}$ LF-NMR relaxation application and supportive methodologies of the following PUFA aggregate structures. A. Non-emulsion formulations, B. Linseed emulsion formulations, and C. Linseed emulsions with fish oil.

Material and Methods:

\section{Samples preparation}

Preparation of linseed emulsion:

Twenty five grams of linseed seeds (Linum usitatissimum ) were grounded and homogenated together with distillated water $(300 \mathrm{ml})$ three times for 10 minutes. The mixture was filtered through gauze and then it was put in the centrifuge $(\mathrm{x} 7000 \mathrm{rpm})$ for $20 \mathrm{~min}$. The top liquid supernatant phase of the mixture was transferred to a new bottle. The bottle was then sonicated on ice for 6 minutes, and after 5 minutes of break it was sonicated again for more 6 minutes.

Preparation of linseed emulsion enriched with fish oil:

Fifty grams of linseed seeds were grounded and homogenated together with distillated water $(720 \mathrm{ml})$. Fish oil $(20 \mathrm{~g})$ was added while mixing in the homogenizer. The mixture was filtered through gauze and then it was put in the centrifuge $(\mathrm{x} 7000 \mathrm{rpm})$ for $20 \mathrm{~min}$. The top liquid supernatant phase of the mixture was transferred to a new bottle. The bottle was then put in the sonicator on ice, sonicated for 6 minutes, and after 5 minutes of break it was sonicated again for more 6 minutes.

\section{Thermal oxidation Procedure}

Thermal oxidation experimental design was based on previous studies (Resende et al., 2020). The samples were autoxidized by heating $100 \mathrm{ml}$ samples in a $250 \mathrm{ml}$ beaker on a hot plate at $55 \mathrm{degC}$. Air was pumped into the beaker with maximum magnetic stirring for 96 hours. Before the induced autoxidation process (time 0 ) and after 24, 48, 72 and 96 hours a $20 \mathrm{ml}$ sample was removed into a vial which was placed in cold water for $10 \mathrm{~min}$ and then stored sealed at $-20 \operatorname{deg} \mathrm{C}$ to avoid further oxidation prior to analysis.

\section{Physical Characterization}

\section{Confocal Laser Scanning Microscope:}

For confocal laser scanning microscopy imaging we used a Zeiss Axio-Observer Z1 inverted microscope, equipped with a Pecon stage-top incubator with temperature, humidity, and $\mathrm{CO}_{2}$ control. The objective used was the 63x Zeiss Plan-Apochromat oil, 1.4 NA, DIC and the imaging was conducted without prior dilution. The samples were excited by a $488 \mathrm{~nm}$ argon-ion laser.

Cryo-SEM specimen preparation and analysis:

For cryogenic scanning electron microscopy (cryo-SEM) imaging: A Zeiss Ultra Plus high-resolution SEM, equipped with a Schottky field-emission gun and with a BalTec VCT100 cold-stage maintained below -145 $\operatorname{deg} \mathrm{C}$. Specimens were imaged at low acceleration voltage of $1.4 \mathrm{kV}$, and working distances of 3-5 mm. We used the Everhart Thornley ("SE2") secondary electron detector.

Specimens were prepared by the drop plunging method: A $3 \mu \mathrm{L}$ drop of solution is set on top of a special planchette maintaining its droplet shape and is manually plunged into liquid ethane, after which it is set on top of a specialized sample table set in a liquid nitrogen pool. The frozen specimens are then mounted on a specialized sample table under liquid nitrogen, and transferred by a high vacuum cryo-transfer shuttle (VCT100; Bal-Tec) to a freeze-fracture system (BAF060; Leica) where it is kept at $-170{ }^{\circ} \mathrm{C}$. In the BAF060, the frozen droplets are fractured by a rapid stroke from a cooled knife, exposing their inner part. They are then transferred into the pre-cooled HR-SEM as described above. Ideally, imaging is performed as close as possible to the drop surface, where cooling rate should be maximal.

Dynamic light scattering (DLS): 
DLS measurements were performed using a CGS-3 light-scattering spectrometer equipped with an LSE-5004 digital correlator (ALV, Langen, Germany). The light source was the He-Ne laser line, $633 \mathrm{~nm}$ at $22 \mathrm{~mW}$. Before the analysis, the samples were diluted about 1/100000 in order to avoid counter saturation and particle interactions (Bouanani et al., 2012).

Zeta potential:

Zeta potential measurements were conducted using a Malvern Panalytical Zetasizer Nano ZS Laser Doppler Micro-electrophoresis instrument. Prior the measurements, the samples were diluted to about 1/100000. Measurements were done in $25^{\circ} \mathrm{C}$.

\section{Chemical Characterization}

$G C-M S$ analysis:

Fatty acids profile of all the samples used in the study was determined before and after thermal heating. The GC-MS analyses were done according to procedure previously described in details by Resende et. al 2020. Gas chromatography-mass spectrometry analyses were performed using an GC-MS system SCION (Bruker, USA), GC-436. Detection was carried out with SQ mass-selective single quadrupole detector. The GC-MS operation control and data process were carried out by Bruker MS Workstation 8 SP2 for SCION software package. The injector temperature was $280 \mathrm{degC}$. The oven temperature was held at $70 \operatorname{degC}$ for $1 \mathrm{~min}$, then increased to $270 \mathrm{degC}$ at a heating rate $7 \mathrm{degC} / \mathrm{min}$. The carrier gas was helium (purity 99.999\%) at flow rate $1.6 \mathrm{ml} / \mathrm{min}$. The sample volume was $1 \mu \mathrm{l}$. The conditions for election impact ionization (EI) were an ion energy of $70 \mathrm{eV}$ and mass range scanning was $39-500 \mathrm{~m} / \mathrm{z}$.

\section{Peroxide Value (PV)}

The primary oxidation products were evaluated with peroxide values (PV) tests according to the AOAC Official Method 965.33.12 (Official methods of analysis of AOAC international, 17th edn. Maryland, USA). Four replicates of each sample were tested.

\section{LF-NMR relaxation}

Signal Acquisition and Data processing of $T_{1}$ (spin-lattice), $T_{2}$ (spin-spin) and $2 D\left(T_{2}-T_{1}\right)$ spectra:

The ${ }^{1} \mathrm{H}$ LF-NMR measurements was carried out on a Maran bench-top pulsed NMR analyzer (Resonance Instruments, Witney, United Kingdom) with a permanent magnet and a18-mm probe head operating at 23.4 MHz. Prior to the measurements, the samples were stabilized at $40{ }^{\circ} \mathrm{C}$ for $40 \mathrm{~min}$ and then allowed to equilibrate inside the instrument for $5 \mathrm{~min}$. The receiver gain (RG) and magnetic field were calibrated before each measurement. The $1 \mathrm{D}$ spectrum of spin-lattice relaxation time $\left(\mathrm{T}_{1}\right)$ was generated using an Inversion Recovery Sequence (INVRC). This sequence consists of a 180 degree radiofrequency pulse (inversion pulse) to the sample followed by a 90 degree radiofrequency pulse. The $1 \mathrm{D}$ spectrum of spin-spin relaxation time $\left(\mathrm{T}_{2}\right)$ was generated using a Carr-Purcell-Meiboom-Gill (CPMG) pulse sequence. The CPMG sequence consists in applying a 90 degree radiofrequency pulse to the sample, followed by many 180 degree pulses. Each time a 180 degree pulse was applied, the signal decay of the magnetic field was removed and a single data point was acquired (Carr and Purcell, 1954; Meiboom and Gill, 1958). The 2D cross-correlation experiments were performed by an Inversion Recovery- CPMG sequence, where the inversion recovery step [180deg- $\mathrm{t}_{1}$ ] is inserted prior to the Carr-Purcell-Meiboom-Gill (CPMG) sequence (Resende et al., 2019ab; Weisman et al., 2018; Song et al. 2002).

The data processing methodology used to reconstruct the signal acquired, into a $2 \mathrm{D}\left(\mathrm{T}_{1}\right.$ vs. $\left.\mathrm{T}_{2}\right)$ spectrum used in this study was developed by our laboratory, as described in previous studies (Berman et al., 2013; Campisi-Pinto et al., 2018; 2019; Weisman et al., 2019; Resende et al., 2019a,b). NMR with low field magnets requires a special data processing methodology in order to maximize the amount of information extracted from the acquired signals. Our methodology uses the primal dual interior method for convex objectives optimization (PDCO) solver and provides an excellent spectrum reconstruction accuracy. 


\section{Self-Diffusion:}

The self-diffusion measurements were carried out with a $20 \mathrm{MHz}$ minispec bench-top pulsed NMR analyzer (Bruker Analytic GmbH, Germany), equipped with a permanent magnet, and a 10-mm temperature controlled probe head. Prior to each measurement, the samples were stabilized at $40 \operatorname{deg}$ for 40 min and then allowed to equilibrate inside the instrument for $5 \mathrm{~min}$ (Meiri et al., 2015). The self-diffusion coefficient, D, was determined by the pulsed-field gradient spin echo (PFGSE) method (Stejskal and Tanner, 1965). The pulse sequence was used with 16 scans, $\tau$ of $7.5 \mathrm{~ms}$, and a recycle delay of $6 \mathrm{~s}$. Typical gradient parameters were $\Delta$ of $7.5 \mathrm{~ms}, \delta$ of $0.5 \mathrm{~ms}$, time between the $90^{\circ}$ pulse to the first gradient pulse of $1 \mathrm{~ms}$, and $G$ of $1.6 \mathrm{~T} / \mathrm{m}$. Each reported self-diffusion coefficient (D) value is the average of ten measurements.

\section{Statistical Analysis}

Statistical analyses were performed using Excel software (Microsoft Office, Windows 7). Three or four replicates of each sample were tested and mean + SD are presented.

\section{Results and discussion:}

A. Characterization of PUFA-rich Linseed oil (LSO) and Fish oil (FO) susceptibility to thermal autoxidation using ${ }^{1} \mathrm{H}$ LF-NMR energy relaxation Time Domain (TD) graphical peak fingerprint material analysis.

To understand the LF ${ }^{1} \mathrm{H}$ NMR analysis of emulsions we first characterized the pure linseed and fish oil. Linseed oil and fish oil are both PUFA-rich materials. Linseed oil is composed mainly of linolenic acid (18:3, n-3; ALA), linoleic acid (18:2, n-6; LA) and oleic acid (18:1, n-9; OA). Fish oil is rich in the omega-3 long chain PUFAs docosahexaenoic acid (22:6, n-3; DHA) and eicosapentaenoic acid (20:5, n3; EPA) (Azuma et al., 2009). PUFAs have biochemical and physiological effects on human health enhancement, but the presence of multiple double bonds and bisallylic carbons affects their oxidative susceptibility, and generation of toxic oxidative products, directly proportional to their degree of unsaturation (Miyashita et al., 1993; 2018).

In the liquid oil phase, fatty acids are self-associated as hydrogen-bonded head to head dimers that are further aggregate as quasi-liquid crystal clusters alternating head to tail arrangement. The cluster aggregates determine the fatty acids liquid properties (Iwahashi and Kasahara, 2011; Berman et al., 2015; Resende et al., 2019b). The dimer fatty acid assembly is characterized by four different segmental motions. The ionic head to head groups are the most rigid microstructure, followed by the double bonds, then aliphatic chain, and the tail chain is the most mobile segment. In the triacylglycerol(TAG) structure, a glycerol bridges 3 FA chains, and is also characterized by four different segmental motions (Resende et al., 2019a)

The ${ }^{1} \mathrm{H}$ LF-NMR energy relaxation TD graphical peak fingerprinting analysis of LSO and FO are shown on Fig. 1 LSO and FO TAG structure is characterized by four different rigidity-mobility segments and each one of these segments have characteristic $\mathrm{T}_{1}$ (spin-lattice) and $\mathrm{T}_{2}$ (spin-spin) energy relaxation times. Analysis of the one-dimensional $\mathrm{T}_{1}$ and $\mathrm{T}_{2}$ spectra of LSO and FO, shows the presence of fours characteristic peaks. From previous studies (Berman et al., 2015; Resende et al., 2019b), peak 1 is assigned to glycerol since the most rigid microstructure and therefore corresponds to the lowest energy relaxation times. Peak 2 is assigned as double bonds as the second most rigid microstructure. Peak 3 is assigned to the aliphatic chains that have less rigidity and peak 4 is assigned as the tail chain as the most mobile segment, corresponding to the longest energy relaxation time.

Thus as described in the above paragraph and shown in Fig. 1 and Table 1 the basic molecular segment pattern analysis by ${ }^{1} \mathrm{H}$ LF-NMR is based on the glycerol segment as the most rigid moiety, followed by the double bonds of the FA chains, the aliphatic chains and aliphatic tails, respectively. In the cases of linseed oil (LSO) and fish oil (FO) of the present study a similar $\mathrm{T}_{1}-\mathrm{T}_{2} \mathrm{TD}(59-55 \mathrm{~ms})$ was assigned to the triacylglycerols head groups of the two oils. Additional three relatively rigid TDs with low mobility peaks were assigned to double bonds (mono-, di- and polyunsaturated fatty acids) with increasing mobility for the two oils (Table 1), in agreement with previous reports (Berman et al., 2015, Meiri at., al 2015). Two peaks 
assigned to the long and short aliphatic chains were also assigned as well as two peaks assigned to the longer tails of omega- 6 and short tail of omega- 3 were identified for the two oils.

(a)Linseed Oil 1D $T_{1}$ and $T_{2}$ spectra
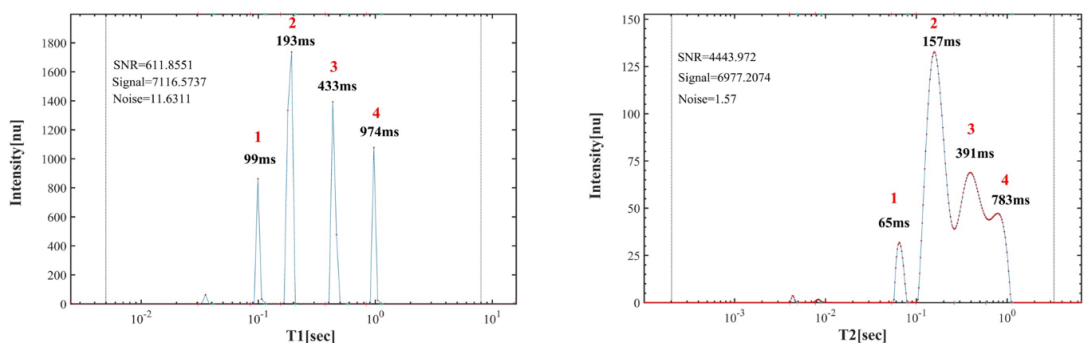

(b) Linseed Oil 2D T1-T2 spectrum

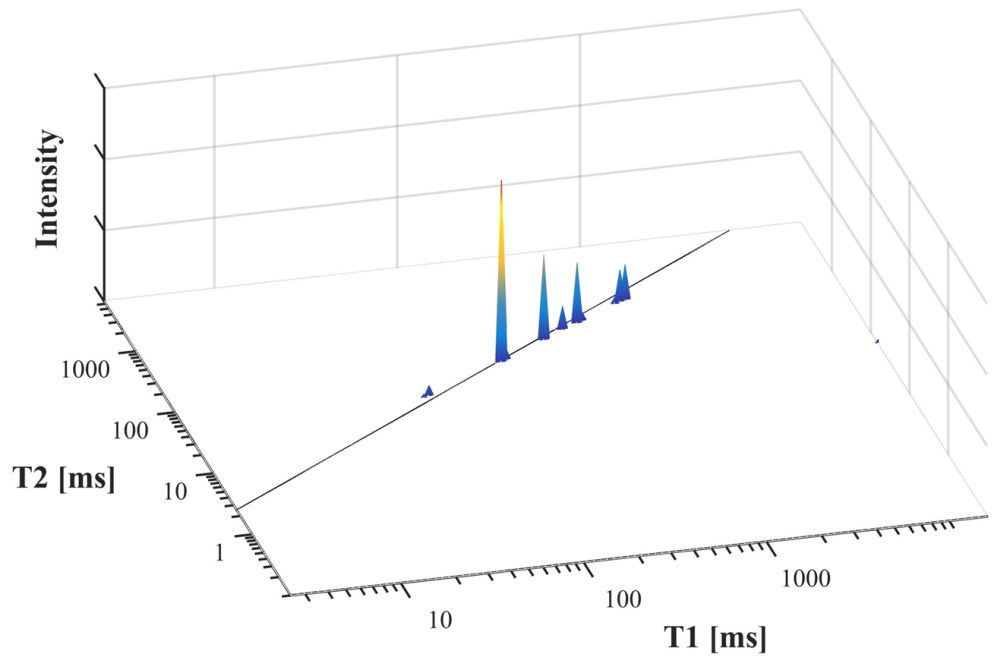

(a) Fish Oil $1 D T_{1}$ and $T_{2}$ spectra
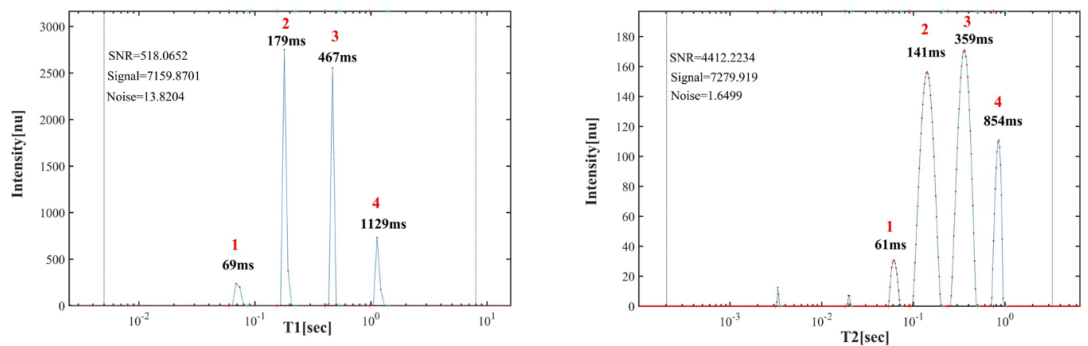
(b) Fish Oil 2D T1-T2 spectrum

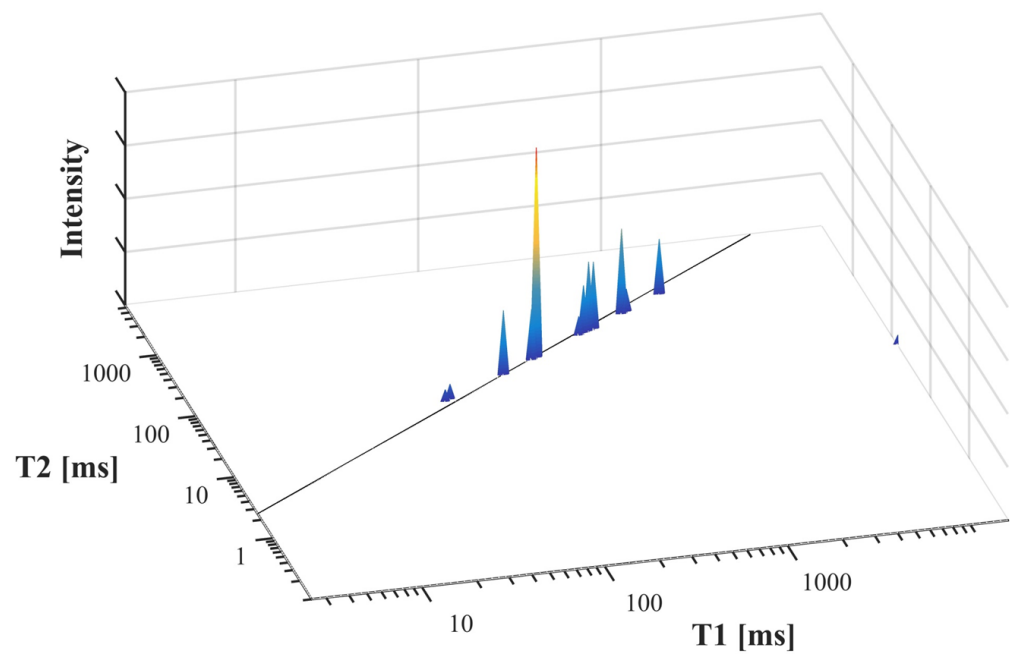

Fig. $1{ }^{1} \mathrm{H}$ LF-NMR energy relaxation time $1 \mathrm{D} \mathrm{T}_{1}$ and $\mathrm{T}_{2}$ and 2D $\mathrm{T}_{1}-\mathrm{T}_{2}$ spectra of linseed oil (LSO, a and b) and fish oil (FO, c and d).

Table 1: $\mathrm{T}_{1}$ and $\mathrm{T}_{2}$ TD values of LSO and FO.

\begin{tabular}{llllll}
\hline & LSO & LSO & FO & FO & \\
\hline Peak & $\mathbf{T}_{\mathbf{1}}$ & $\mathbf{T}_{\mathbf{2}}$ & $\mathbf{T}_{\mathbf{1}}$ & $\mathbf{T}_{\mathbf{2}}$ & ${ }^{\mathbf{1}} \mathbf{H}$ TD assignment \\
1 & 1129 & 798 & 1408 & 1036 & FA - Tail (omega-3) \\
2 & 1049 & 764 & 781 & 602 & FA - Tail (omega-6) \\
3 & 582 & 454 & 502 & 399 & FA - Aliphatic (shorter chains) \\
4 & 541 & 425 & 467 & 374 & FA - Aliphatic (longer chains) \\
5 & 433 & 358 & 433 & 358 & FA - D. Bonds (poly) \\
6 & 323 & 270 & 208 & 182 & FA - D. Bonds (di) \\
7 & 166 & 147 & 124 & 113 & FA - D. Bonds (mono) \\
8 & 59 & 55 & 59 & 55 & Glycerol \\
\hline
\end{tabular}

In our previous studies that have demonstrating ${ }^{1} \mathrm{H}$ LF-NMR energy relaxation time chemical and morphological spectral mapping as an efficient tool to characterize LSO's thermal oxidation (Resende et al., 2019a,b). In the present study for comparison to their emulsions, both LSO and FO were thermal oxidized with air at $55^{\circ} \mathrm{C}$ for 96 hours and their $2 \mathrm{D}$ TD fingerprint map are shown respectively at Fig. 2.

(a)

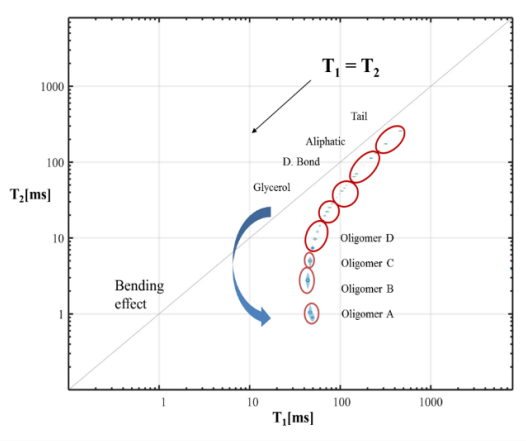

(b)

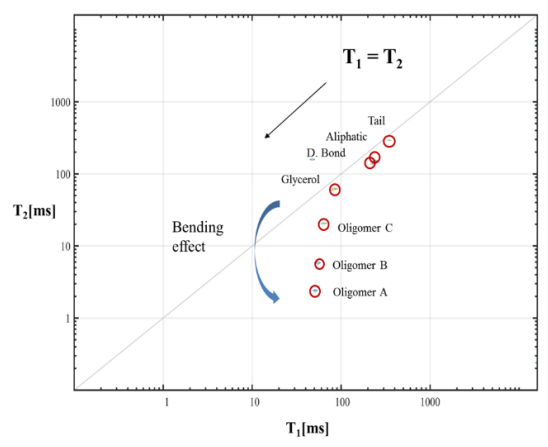


Fig. 2 Demonstration of LSO (a) and FO (b) $2 \mathrm{D}^{\mathrm{T}} \mathrm{T}_{1}-\mathrm{T}_{2}$ TD fingerprinting map after $96 \mathrm{~h}$ heating at $55{ }^{\circ} \mathrm{C}$.

The LSO and FO ${ }^{1} \mathrm{H}$ LF NMR energy relaxation time spectra after undergoing oxidation (Fig. 2) has more peaks than the four characteristic peaks of the original LSO and FO (Fig. 1). These new peaks correspond to the oxidation products. The spectrum of a non-oxidized oil (Fig. 1) is characterized by 4 major peaks with the $T_{1}$ and $T_{2}$ of similar values with the peaks being aligned in the diagonal of the map. After oxidation the map of Fig. 2 not only present new peaks related to the oxidation products but there is also a bending effect in the curve from the diagonal because of decreasing $\mathrm{T}_{2}$ values, indicating oxidation (Resende et al., 2019a,b). In order to confirm the oxidation of the LSO and FO after 96 hours of thermal oxidation observed in the ${ }^{1} \mathrm{H}$ LF-NMR energy relaxation time map of Fig. 2, these material were also analyzed by peroxide value (PV) tests. The PV test results (Table 2) support the oxidation of the LSO and FO wherein the fish oil shows a higher PV value, indicating a higher degree of oxidation than the LSO. This is in agreement with the GC-MS results of changes of fatty acids profile during thermal heating (not shown) and previously published literature (Resende et al., 2019), because of the higher number of double bonds in the fish oil increase its propensity to oxidation.

Table 2: Peroxide values (PV) of LSO and FO in thermal conditions $\left(55^{\circ} \mathrm{C}\right)$

\begin{tabular}{lll}
\hline & T-0 & T-96 \\
\hline LSO & $2.77+0.42$ & $156.92+27.56$ \\
FO & $4.53+0.65$ & $404.60+71.04$ \\
\hline
\end{tabular}

B. Characterization of linseed emulsions (LSE) and Linseed fish oil (LSFE) emulsion mixtures oxidative stability and structural changes under thermal oxidizing conditions $\left(55^{\circ} \mathrm{C}\right) \mathrm{using}^{1} \mathrm{H}$ LF-NMR energy relaxation time application and supportive methodologies.

Fig. 3 shows visual images of linseed emulsion (LSE) and linseed emulsion enriched with fish oil (LSFE) in comparison to pure linseed oil (LSO), and fish oil (FO) of the present study. The visual characterization show that the emulsions are homogenous and physically stable without phase separation. Fig. $4 \mathrm{a}$ and $\mathrm{c}$ are microscopy images of LSE and LSFE respectively showing the supramolecular assembly of the fatty acid oils as oil bodies. Fig. 4 b1, b2 and d1, d2 are the Cryo-SEM images for LSE and LSFE respectively, that show in a good resolution the structure of the oil bodies in the emulsions.

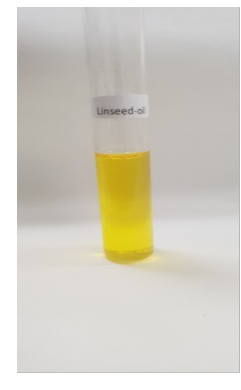

LSO

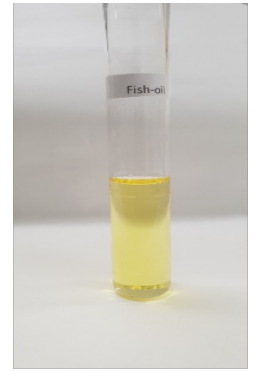

FO

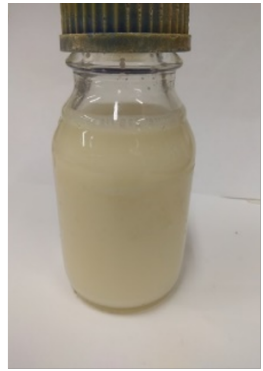

LSE

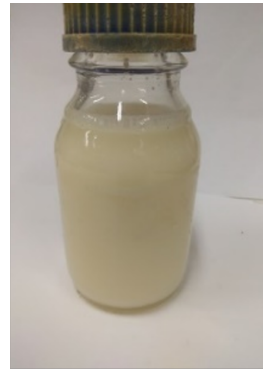

LSFE

Fig. 3 Visual images of oils: Linseed Oil (LSO) and Fish Oil (FO) and oil bodies of linseed emulsion (LSE) and linseed enriched with fish oil emulsion (LSFE) 


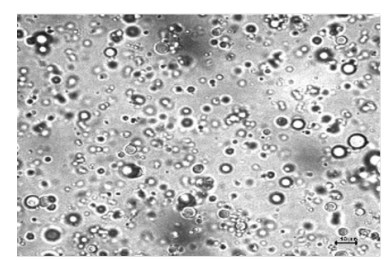

(a)

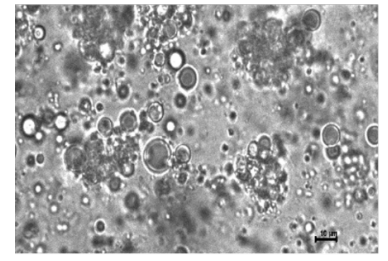

(c)

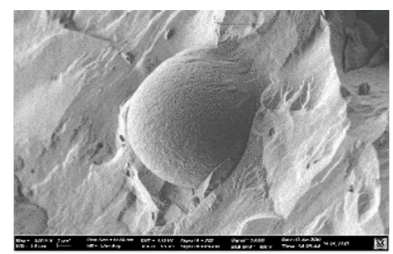

(b1)

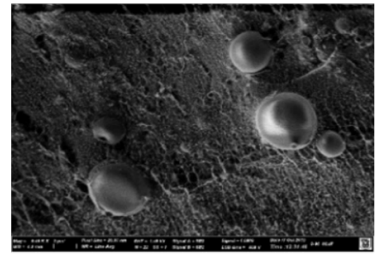

(d1)

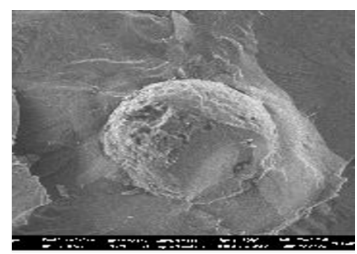

(b2)

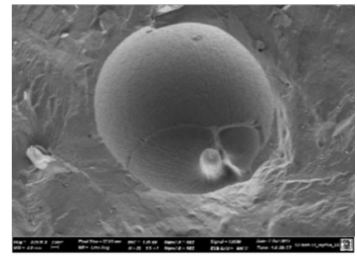

(d2)

Fig. 4 Microscopic images of LSE and LSFE. (a \& c) Confocal oil bodies image; (b1 \& b2) Cryo-SEM intact LSE oil bodies; (d1 \& d2) Cryo-SEM LSFE oil body.

In the linseed emulsion (LSE) and linseed fish oil enriched emulsion (LSFE) of this study, the supramolecular assembly of the lipid molecules is characterized by the oil body's (OB) physical and chemical aggregated organization. This structural organization contains polar head groups forming the outside as the surface of the oil bodies in contact with water, while the hydrophobic tails are inside and away from the aqueous matrix. The OB's interfacial surface coating layer contains the LS's phospholipids and amphiphilic proteins such as oleosins and residual linseed mucilage components, that together stabilize the OB (Goyal et al., 2015; Fabre et al., 2015). Thus LSE chemical and morphological structures are oil body vesicles with a multicomponent amphiphilic surface and lipophilic core of encapsulated PUFA-rich oil

The ${ }^{1} \mathrm{H}$ LF-NMR energy relaxation TD fingerprinting for LSE before and after thermal oxidation at $55{ }^{\circ} \mathrm{C}$ for 96 hours are shown in Fig. 5. This figure shows that before LSE oxidation the oil's $\mathrm{T}_{1}$ and $\mathrm{T}_{2}$ energy relaxation time consists of two different parts. One is related to the PUFA-rich oil molecules within the OB's core and the other on the OB's interfacial surface. The $T_{1}$ and $T_{2}$ energy relaxation time of the oil body's vesicle surface is not significantly affected by the water because the oil's energy relaxation time rate is much faster than water's energy relaxation time (Kleinberg et al., 1994). The water $\mathrm{T}_{1}$ and $\mathrm{T}_{2}$ energy relaxation time rates are primarily within the continuous bulk water phase. The high $\mathrm{T}_{1}$ and $\mathrm{T}_{2}$, of bulk water are out of the range of the ${ }^{1} \mathrm{H}$ LF-NMR energy relaxation graphics of Fig. 5.

It is interesting that in both samples of LSE before and after thermal oxidation at $55{ }^{\circ} \mathrm{C}$ for 96 hours very minimal phase separation is observed (see supplemental 1). Similarly in Fig. 5 a similar peak pattern/arrangement is seen on the ${ }^{1} \mathrm{H}$ LF-NMR energy relaxation TD graphics of LSE before and after thermal oxidation at $55{ }^{\circ} \mathrm{C}$ for 96 hours. Both samples before and after oxidation, have a $1 \mathrm{D} \mathrm{T}_{1}$ energy relaxation time spectrum characterized by three peaks corresponding to the oil molecules in the core of the vesicles and one peak of higher intensity related to oil's PUFA energy relaxation in the vesicle's surface volume. Their 1D $\mathrm{T}_{2}$ energy relaxation time spectrum has four small peaks assigned to the four segmental motions of the oil molecules in the core of the oil body's vesicles and one peak of higher intensity related to oil's PUFA energy relaxation in the vesicle surface. The 2D energy relaxation time spectrum of LSE $(\mathrm{T} 0 \mathrm{~h})$ and LSE $(\mathrm{T} 96 \mathrm{~h})$ presents a peak of high intensity assigned to the energy relaxation of the oil bodies vesicle's surface and small peaks assigned to the PUFA-rich oil molecules in the core of the oil bodies (Fig. 5, Table 3). Although the pattern of the 1D $\mathrm{T}_{1}$ and $\mathrm{T}_{2}$ and $2 \mathrm{D}$ relaxation time graphics of LSE (T0 h) and LSE (T 96 $\mathrm{h}$ ) are very similar, the analysis of the $\mathrm{T}_{1}$ and $\mathrm{T}_{2}$ peaks values presented in Table 3 showed that the sample of LSE after T $96 \mathrm{~h}$ oxidation has somewhat lower relaxation times than the sample of fresh LSE at T $0 \mathrm{~h}$, most probable due to the thermal oxidation process and resulting increasing internal viscosity. 
Average droplet particles size distribution as determined by dynamic light scattering (DLS) system shows an increase of oil bodies size from $803.2 \mathrm{~nm}$ at T $0 \mathrm{~h}$ to $1363 \mathrm{~nm}$ after T $96 \mathrm{~h}$ of thermal heating (Table 4). Microscopic images of fresh LSE sample versus LSE sample after $96 \mathrm{~h}$ of thermal heating further demonstrate this observation (see in supplemental material section). Indeed this pattern of vesicles size increase is well explained by a process of vesicles fusion after addition of heat energy (Millichip et al., 1996; Nikiforidis, 2019). However, this relatively small increase in the average oil bodies size may be correlated with the results of a small reduction in $\mathrm{T}_{1}$ and $\mathrm{T}_{2}$ proton energy relaxation times shown in Fig. 5 due to oxidation, which may indicate that the oil bodies emulsion preparation may be further improved to enhance oxidative stability. Furthermore the results of zeta potential assay show values of -29.1 and -28.7 for $\mathrm{T} 0 \mathrm{~h}$ and $\mathrm{T}$ $96 \mathrm{~h}$, respectively. Usually it is accepted that emulsion preparations with values of zeta potential above -40 are considered as very stable and values of about -30 are considered to represent moderate stability (Nikovska, 2012; Chanamai and McClements 2002). Testing of self-diffusion of LSE samples before and after thermal heating for $96 \mathrm{~h}$ show some minimal decrease from 2.750 to $2.38310^{\wedge}-9 \mathrm{~m} * \mathrm{~m} / \mathrm{s}$, in respectively. These supportive results are also well correlate with previous data described above for linseed oil. The ratio of $T_{1} / T_{2}$ that is taken directly from the $T_{1}-T_{2} T D$ fingerprinting map show the similar pattern of a relative small change from 2.10 to 2.82 for fresh and $96 \mathrm{~h}$ heated LSE samples. This change in $T_{1} / \mathrm{T}_{2}$ ratio values under oxidative conditions represent the chemical and structural changes effects on the signals obtained from the ${ }^{1} \mathrm{H}$ LF-NMR that may be used as a fast tool to monitor the oxidation of the multicomponent complex emulsion samples.

(a) LSE T-0 1D $T_{1}$ and $T_{2}$ spectra
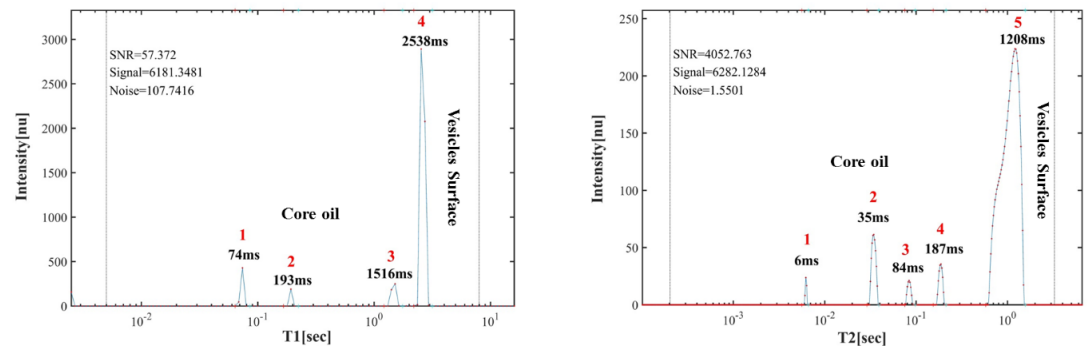

(b) LSE T-0 2D T1-T2 spectrum

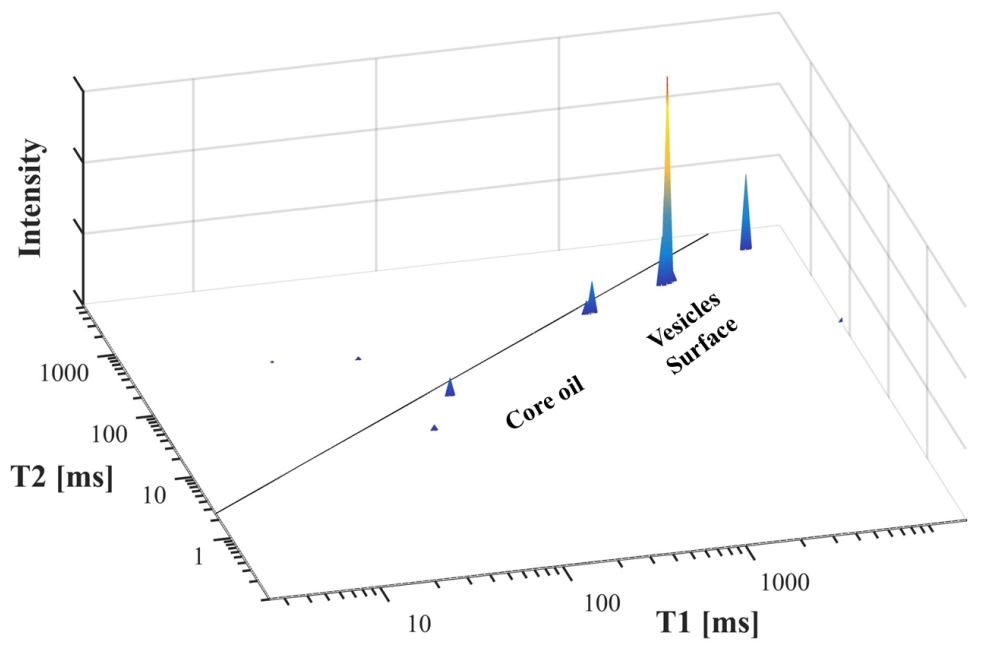



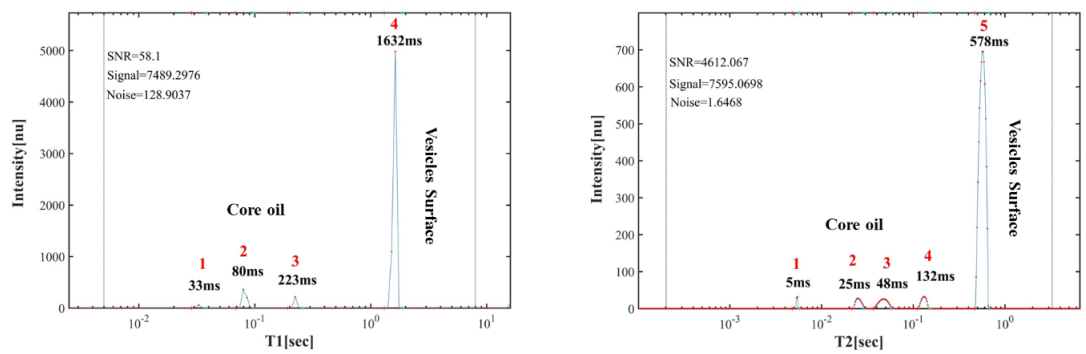

(b) LSE T-96 h 2D T1-T2 spectrum

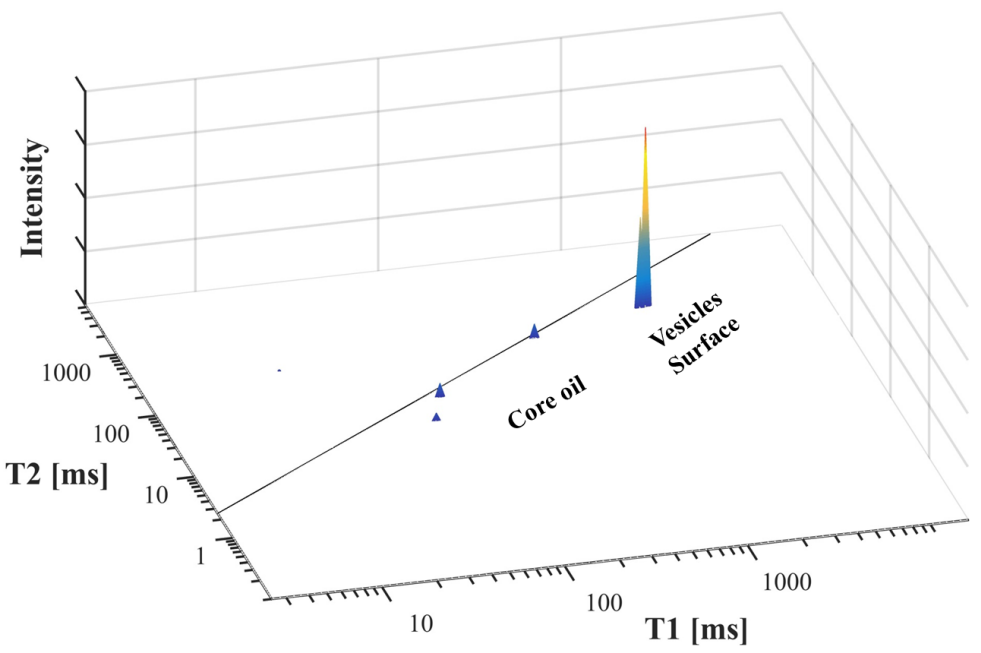

Fig. $5{ }^{1} \mathrm{H}$ LF-NMR relaxation spectra of LSE fresh produced (T-0h, a and b) and after 96 hours of thermal oxidation at $55{ }^{\circ} \mathrm{C}(\mathrm{T}-96 \mathrm{~h}, \mathrm{c}$ and $\mathrm{d})$.

Table 3: ${ }^{1} \mathrm{H}$ LF-NMR energy relaxation time spectra of LSE fresh produced (T $0 \mathrm{~h}$ ) and after 96 hours of thermal oxidation at $55{ }^{\circ} \mathrm{C}\left(\mathrm{T}\right.$ 96h) 2D T1-T2 peaks values and proposed ${ }^{1} \mathrm{H}$ TD assignment.

\begin{tabular}{llllll}
\hline & T-0 & T-0 & T-96 & T-96 & \\
\hline Peak & $\mathbf{T}_{\mathbf{1}}$ & $\mathbf{T}_{\mathbf{2}}$ & $\mathbf{T}_{\mathbf{1}}$ & $\mathbf{T}_{\mathbf{2}}$ & ${ }^{\mathbf{1}} \mathbf{H}$ TD assignment \\
1 & 2941 & 1287 & - & - & Surface (small OB) \\
2 & 2732 & 1206 & 1756 & 615 & Surface (medium OB) \\
3 & 905 & 577 & 374 & 307 & FA - Aliphatic chains \\
4 & 92 & 55 & 80 & 57 & FA - D. Bonds \\
5 & 59 & 18 & 64 & 25 & GLY \\
\hline
\end{tabular}

Table 4: Physical-structural properties of non-heated (T-0) and heated (T-96) LSE

\begin{tabular}{lll}
\hline & T-0 & T-96 \\
\hline $\mathrm{T}_{1} / \mathrm{T}_{2}$ & 2.10 & 2.82 \\
Self diffusion $\left(10^{\wedge}-9 \mathrm{~m} * \mathrm{~m} / \mathrm{s}\right)$ & $2.750+0.018$ & $2.383+0.033$
\end{tabular}




\begin{tabular}{lll}
\hline & T-0 & T-96 \\
\hline DLS $(\mathrm{nm})$ & $803.2+78$ & $1363+84$ \\
Z Potential (mv) & $-29.1+5.3$ & $-28.7+6.5$ \\
\hline
\end{tabular}

\section{Characterization of linseed fish oil emulsions (LSFE) oxidative stability and structural changes during thermal oxidation, using H1 LF-NMR energy relaxation time analysis and supportive methodologies.}

LSFE was prepared, as described in the Material and Methods section by the addition of fish oil rich in the long chain PUFAs EPA and DHA. The chemical and morphological structure of LSFE is characterized by oil bodies vesicles with an amphiphilic interface surface and lipophilic core encapsulating PUFA-rich linseed and fish oil. The ${ }^{1} \mathrm{H}$ LF-NMR energy relaxation TD fingerprinting for LSFE before (T 0) and after thermal oxidation at $55{ }^{\circ} \mathrm{C}$ for 96 hours $(\mathrm{T} 96 \mathrm{~h}$ ) are shown in Fig. 6.

It is interesting to note that before and after thermal oxidation at $55^{\circ} \mathrm{C}$ for 96 hours only minimal phase separation could be observed in the LSFE samples (see supplemental 1). The analysis of Fig. 6 shows that LSFE T $0 \mathrm{~h}$ and LSFE T $96 \mathrm{~h}$ have similar characteristics $\mathrm{T}_{1}$ and $\mathrm{T}_{2}$ energy relaxation time graphics. The 1D $\mathrm{T}_{2}$ energy relaxation time graphics of LSFE T $0 \mathrm{~h}$ and LSFE T $96 \mathrm{~h}$ shows fours small peaks corresponding to the four segmental motions of the PUFA-rich oil molecules within the core of the oil bodies emulsion and one peak of higher intensity related to the oil energy relaxation time in the surface of the oil bodies vesicles. The $2 \mathrm{D} \mathrm{T}_{1}-\mathrm{T}_{2}$ relaxation time graphics of LSFE $\mathrm{T} 0 \mathrm{~h}$ and LSFE $\mathrm{T} 96 \mathrm{~h}$ shows small peaks assigned as the PUFA-rich oil molecules within the OB core and one peak of higher intensity related to the energy relaxation time in the OB's vesicle surface.

In Table 5 the $\mathrm{T}_{1}$ and $\mathrm{T}_{2}$ energy relaxation times values of LSFE $\mathrm{T} 0 \mathrm{~h}$ and LSFE $\mathrm{T} 96 \mathrm{~h}$ are tabulated. It can be seen that the relaxation time values of $T_{1}$ and $T_{2}$ of the main peak assigned as the vesicles surface are somewhat lower for LSFE after the thermal oxidation of 96 hours as are most of the other peaks that could be observed. These data are in the same general pattern of the results obtained for LSE described above (Table 3 ).

Microscopic images of LSFE oil bodies emulsion of non-heated fresh sample and after $96 \mathrm{~h}$ of thermal heating (Fig. 7), show some increase of oil bodies after the heating period. Interesting to note that in both cases before and after heating only minimal phase separation could be observed in the LSFE samples. A similar pattern for LSFE (Table 6), some increase of droplet particle size distribution from 1374 to 1951 $\mathrm{nm}$ for fresh and heated sample, respectively was obtained. Zeta potential values were -27.3 and $-25.1 \mathrm{mv}$ for the two samples, suggested of a moderate rate of stability of the emulsion samples. The rate of self diffusion was slightly reduced from 2.902 to $2.73410^{\wedge}-9 \mathrm{~m} * \mathrm{~m} / \mathrm{s}$, respectively. All these supportive analyses well correlated with the $\mathrm{T}_{1}-\mathrm{T}_{2}$ energy relaxation time information obtained from NMR analyses (Fig. 6) that is also summarized in the small increase of $\mathrm{T}_{1} / \mathrm{T}_{2}$ ratio.

(a) LSFE T-0 1D $T_{1}$ and $T_{2}$ spectra
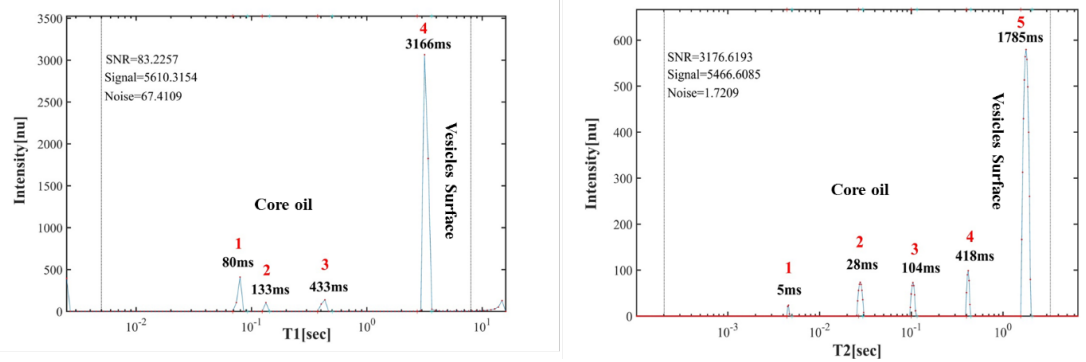
(b) LFSE T-0 2D T1-T2 spectrum

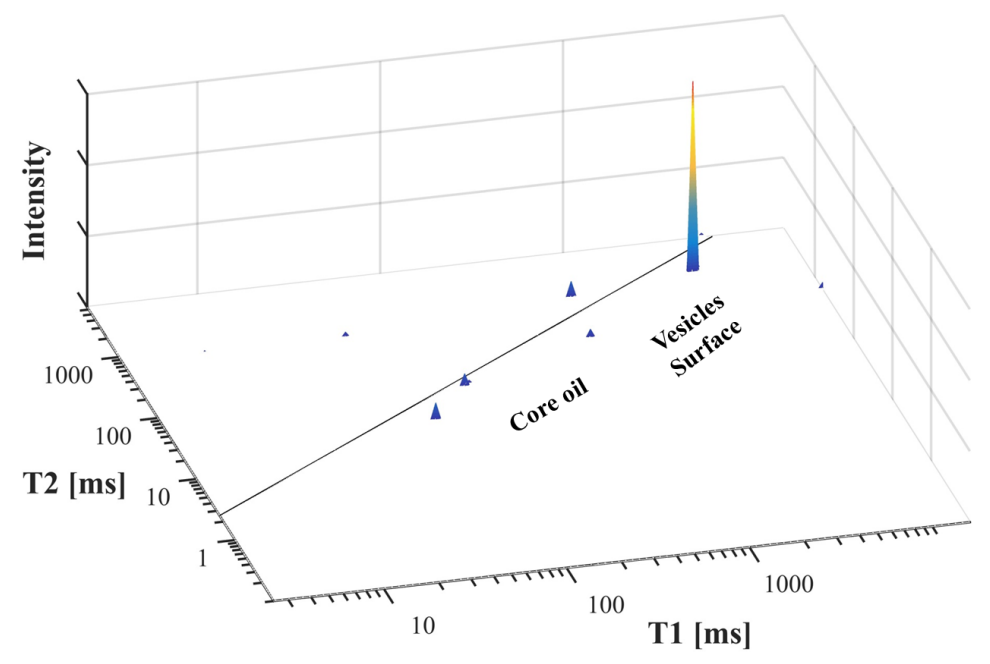

(a)LSFE T-96 $\mathrm{h}$ 1D $\mathrm{T}_{1}$ and $\mathrm{T}_{2}$ spectra
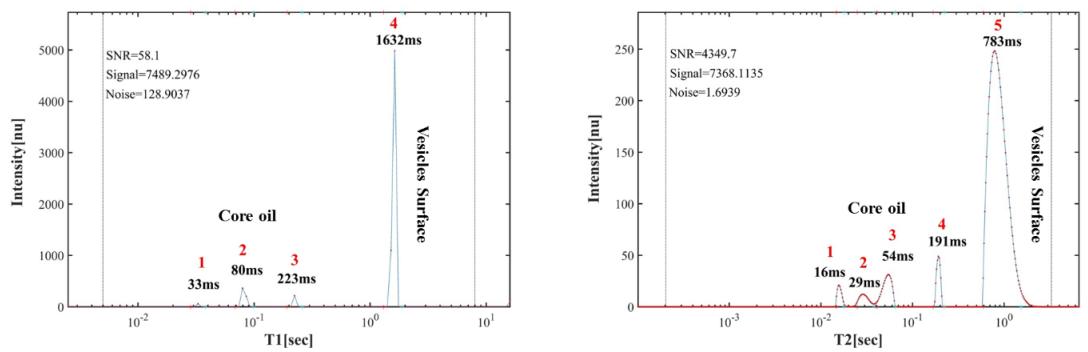

(b) LFSE T-96 h 2D T1-T2 spectrum

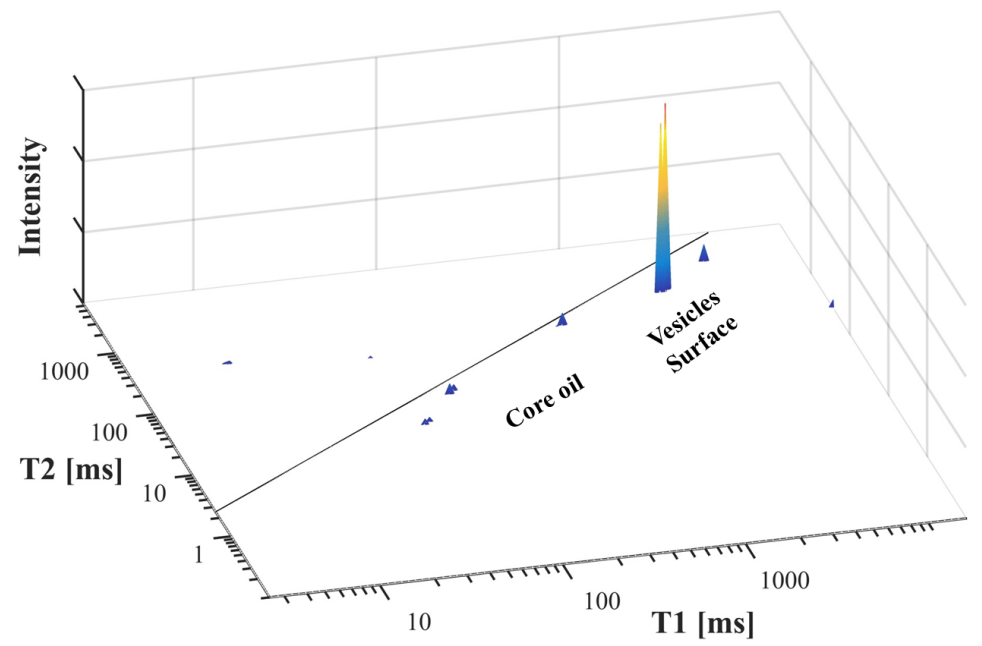

Fig. $6{ }^{1} \mathrm{H}$ LF-NMR relaxation spectra of LSFE fresh produced (T-0h, a and b) and after 96 hours of thermal oxidation at $55{ }^{\circ} \mathrm{C}(\mathrm{T}-96 \mathrm{~h}, \mathrm{c}$ and d). 
Table 5: ${ }^{1} \mathrm{H}$ LF-NMR relaxation spectra of LSFE oil bodies emulsion non-heated (T-0) and heated (T-96) $2 \mathrm{D}$ T1-T2 peaks values and proposed ${ }^{1} \mathrm{H}$ TD assignment.

\begin{tabular}{llllll}
\hline & T-0 & T-0 & T-96 & T-96 & \\
\hline Peak & $\mathbf{T}_{\mathbf{1}}$ & $\mathbf{T}_{\mathbf{2}}$ & $\mathbf{T}_{\mathbf{1}}$ & $\mathbf{T}_{\mathbf{2}}$ & $\mathbf{1}_{\mathbf{H}} \mathbf{T D}$ assignment \\
1 & 3948 & 1902 & 2538 & 971 & OB surface \\
2 & 726 & 282 & 583 & 416 & FA - Aliphatic chains \\
3 & 115 & 84 & 92 & 57 & FA - D. Bonds \\
4 & 64 & 29 & 55 & 21 & Glycerol \\
\hline
\end{tabular}
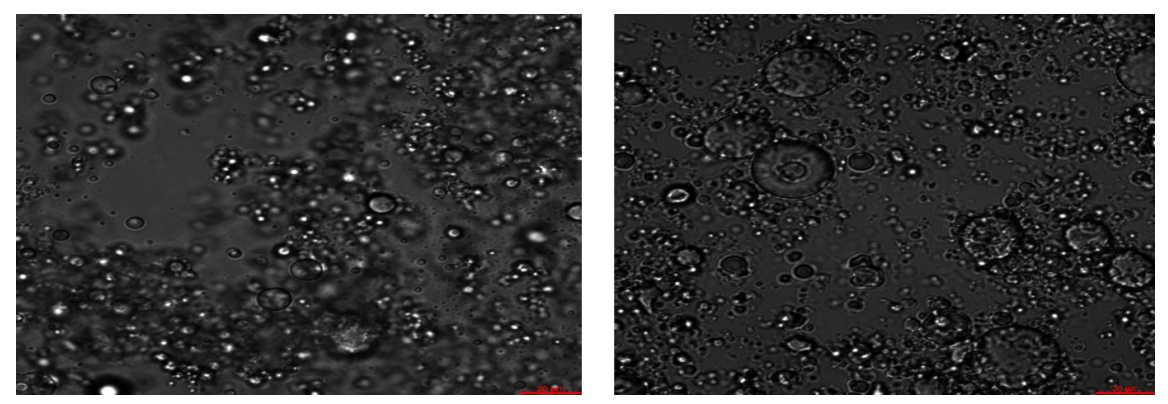

Fig. 7 Microscopic images of LSFE oil bodies emulsion of non-heated sample (left- T-0) and after $96 \mathrm{~h}$ of thermal heating $\left(55^{\circ} \mathrm{C}\right)$ (right T-96)

Table 6: Physical-structural properties of non-heated and heated LSE

\begin{tabular}{lll}
\hline & T-0 & T-96 \\
\hline $\mathrm{T}_{1} / \mathrm{T}_{2}$ & 2.07 & 2.61 \\
Self diffusion $\left(10^{\wedge}-9 \mathrm{~m} * \mathrm{~m} / \mathrm{s}\right)$ & $2.902+0.020$ & $2.7338+0.025$ \\
DLS (nm) & $1374+81$ & $1951+89$ \\
Z Potential (mv) & $-27.3+6.6$ & $-25.1+8.3$ \\
\hline
\end{tabular}

The two oil bodies emulsion preparations of LSE and LSEF used in the present study were analyzed by the conventional peroxide value (PV) test (Table 7). As expected and in agreement with previous reports (Goyal et al., 2015), the two fresh oil bodies emulsions ( $\mathrm{T} 0 \mathrm{~h}$ ) show a low PV value of about $3 \mathrm{meq} / \mathrm{kg}$. After 96 $\mathrm{h}$ of thermal heating conditions of $55^{\circ} \mathrm{C}$ only a very small increase of $\mathrm{PV}$ of about $5 \mathrm{meq} / \mathrm{kg}$ was obtained for the two oil body emulsions. This data is also supported by the GC-MS results showing minimal changes of fatty acids profile of LSE and LSFE during thermal heating (not shown). These PV results well correlate with all the previous data in this study. Furthermore, this small increase of PV may confirm the ability of rapid non-destructive NMR energy relaxation time sensor application to monitor oxidation in complex materials as demonstrated in the present study. In addition considering the reports of emulsion preparation stability at low refrigerated conditions $\left(4-7^{\circ} \mathrm{C}\right)$ (Goyal et al., 2015), the natural linseed oil bodies emulsion preparations encapsulation PUFA-rich components including fish oil shown seems to be relatively stable with high oxidant resistance even under high thermal oxidizing conditions.

Table 7 : Peroxide values $\left(\mathrm{PV}, \mathrm{meqO}_{2} / \mathrm{kg}\right)$ of fresh LSE and LSFE and after thermal conditions $\left(55^{\circ} \mathrm{C}\right)$.

\begin{tabular}{lll}
\hline & T-0 & T-96 \\
\hline LSE & $2.95+0.59$ & $5.24+0.90$
\end{tabular}




\begin{tabular}{lll}
\hline & T-0 & T-96 \\
\hline LSFE & $3.41+0.37$ & $4.98+0.71$ \\
\hline
\end{tabular}

In summary, the capability of ${ }^{1} \mathrm{H}$ LF-NMR energy relaxation time sensor to monitor the TD fingerprint peaks in 2D graphics of chemical and structural changes of linseed oil bodies emulsion (LSE) and linseed emulsion enriched with fish oil (LSFE) in thermal autoxidation conditions was demonstrated. Furthermore, the efficacy of LSE as autoxidation protected fish oil PUFA-rich delivery system was shown.

\section{Acknowledgments:}

The authors would like to acknowledge Dr. Z. Abramovich and Mrs. Shoshana Kravchik for the technical work and also all the members of PLBL for general laboratory assistance.

\section{Conflict of Interest:}

The authors declare that they have no conflict of interest.

Supplemental materials:

Supplemental 1:

T-0 T-96

\section{T-0 T-168}

Fig. A Images of linseed oil bodies emulsion preparation non-heated sample (T-0) and after $96 \mathrm{~h}$ (T-96) of thermal heating $\left(55^{\circ} \mathrm{C}\right)$ plus air pumping (Upper level).

Images of linseed enriched with fish oil oil bodies emulsion preparation. Non-heated sample (T-0) and after $96 \mathrm{~h}(\mathrm{~T}-96)$ of thermal heating $\left(55^{\circ} \mathrm{C}\right)$ plus air pumping (Upper level).

Supplemental 2:

Fig. B Microscopic images of LS Milk oleosomes emulsion non-heated sample (left- T-0) and after $96 \mathrm{~h}$ of thermal heating $\left(55^{\circ} \mathrm{C}\right)$ plus air pumping (right T-96)

\section{References}

Abozid, M. M., \& Ayimba, E. (2014) Effect of omega 3 fatty acids family in human health. International Journal of Advanced Research ,2:202-211.

Azuma, G., Kimura, N., Hosokawa, M., \& Miyashita, K. (2009) Effect of Droplet Size on the Oxidative Stability of Soybean Oil TAG and Fish Oil TAG in Oil-in-Water Emulsion. Journal of Oil Science, 58 : 329-338

Bakota, E. L., Winkler-Moser, J.K., \& Palmquist, D. E. (2012) Solid fat content as a substitute for total polar compound analysis in edible oils. Journal of the American Oil Chemists' Society, 89:2135-2142.

Berman, P., Levi, O., Parmet, Y., Saunders, M., \& Wiesman, Z. (2013) Laplace inversion of low-resolution NMR relaxometry data using sparse representation methods. Concepts in Magnetic Resonance Part A ,42 :72-88.

Berman, P., Meiri, N., Colnago, L. A., Morales, T. B., Linder, C., Levi, O., ... Wiesman, Z. (2015) Study of liquid-phase molecular packing interactions and morphology of fatty acid methyl esters (biodiesel).Biotechnology for Biofuels , $8: 12-28$.

Blumich, B. (2016) Introduction to compact NMR: A review of methods. Trends in Analytical Chemistry, 83 $: 2-11$. 
Bouanani, F., Bendedouch, D., Teixeira, J., Marx, L., Hemery, P. (2012) Characterization of a miniemulsion by DLS and SANS. Colloids and Surfaces A: Physicochemical and Engineering Aspects, 404: $47-51$

Campisi-Pinto, S., Levi, O., Benson, D., Cohen, M., Resende, M. T., Saunders, M., \& Wiesman, Z. (2018) Optimal regularization parameters of primal-dual interior method for convex objectives (PDCO) applied to 1H low field nuclear magnetic resonance (1H LF-NMR). Applied Magnetic Resonance , 49 :1129-1150.

Campisi-Pinto, S., Levi, L., Benson, D., Resende, M. T., Saunders, M., Linder, C., \& Wiesman, Z. (2019) Simulation-Based Sensitivity Analysis of Regularization Parameters for Robust Reconstruction of Complex Material's T1 - T21H LF-NMR Energy Relaxation Signals. Applied Magnetic Resonance , 1-18.

Carr, H. Y., \& Purcell, E. M. (1954) Effects of diffusion on free precession in nuclear magnetic resonance experiments. Physical Review , 94 :630-638.

Chanamai, R., \& McClements, D. J. (2002) Comparison of Gum Arabic, Modified Starch, and Whey Protein Isolate as Emulsifiers: Influence of pH, CaCl2 and Temperature. Journal of Food Science, 67 :120 - 125.

Fabre, J.-F., Lacroux E., Cerny, M., \& Mouloungui, Z. (2015) Barriers to the release of flaxseed oil bodies and ways of overcoming them. Oilseeds and fats crops and lipids, 22 D607: 1-7.

Fisk, I. D., White, D. A., Lad, M., \& Gray, D. A. (2008) Oxidative stability of sunflower oil bodies. European Journal of Lipid Science and Technology, 110 : 962-968.

Goyal, A., Sharma, V., Upadhyay, N., Singh, A. K., Arora, S., Lal, D., \& Sabikhi, L. (2015). Development of stable flaxseed oil emulsions as a potential delivery system of $\omega-3$ fatty acids. Journal of food science and technology , 52 : 4256-4265. https://doi.org/10.1007/s13197-014-1370-2

Guillén, M. D., \& Uriarte, P. S . (2009) Contribution to further understanding of the evolution of sunflower oil submitted to frying temperature in a domestic fryer: study by $1 \mathrm{H}$ nuclear magnetic resonance. Journal of Agricultural and Food Chemistry, $\mathbf{5 7}: 7790-7799$.

Gouilleux, B., Charrier, B., Akoka, S., Felpin, F-X., Rodriguez-Zubiri, M., \& Giraudeau, P. (2016) Ultrafast 2D NMR on a benchtop spectrometer: Applications andperspectives. Trends in Analytical Chemistry ,83 : $65-75$

Huang, A. H. C. (1994) Structure of Plant Seed Oil Bodies. Current Opinion in Structural Biology , 4 :493-498.

Humar, M., \& Lesar, B. (2013) Efficacy of linseed- and tung-oil-treated wood against wood-decay fungi and water uptake. International Biodeterioration 83 Biodegradation , 85:223-227.

Hwang, H. S., Winkler-Moser, J. K. \& Liu, S. (2017) Reliability of 1H NMR Analysis for Assessment of Lipid Oxidation at Frying Temperatures. Journal of the American Oil Chemists' Society,94: 257-270.

Iwahashi, M., \& Kasahara, Y. (2011) Dynamic molecular movements and aggregation structures of lipids in a liquid state. Current Opinion in Colloid \& Interface Science, 16 : 359-366.

Jacobsen, C. (2015) Some strategies for the stabilization of long chain n-3 PUFA-enriched foods: A review. European Journal of Lipid Science and Technology, 117 : 1853-1866.

Jackowski, S. A., Alvi, A. Z., Mirajkar, A., Imani, Z., Gamalevych, Y., Shaikh, N. A., \& Jackowski, G. (2015) Oxidation levels of North American over-the-counter n-3 (omega-3) supplements and the influence of supplement formulation and delivery form on evaluating oxidative safety. Journal of Nutritional Science, 4:1-10.

Jhala, A., \& Hall, L. M. (2010) Flax (Linum usitatissimum L.): Current uses and future applications. Australian Journal of Basic and Applied Sciences , 4 :4304-4312

Jolivet, P., Boulard, C., Bellamy, A., Larre, C., Barre, M., Rogniaux, H., D'andrea, S., Chardot, T., \& Nesi, N. (2009) Protein Composition of Oil Bodies from Mature Brassica napus Seeds. Proteomics ,9 : 3268-3284. 
Karefyllakis, D., Jan Van Der Goot, A., \& Nikiforidis, C. V. (2019). The behaviour of sunflower oleosomes at the interfaces. Soft Matter , 15 : 4639-4646. https://doi.org/10.1039/c9sm00352e

Meiboom, S., \& Gill, D. (1958) Modified spin-echo method for measuring nuclear relaxation times. Review of Scientific Instruments ,29 : 688-691.

Meiri, N., Berman, P., Colnago, L. A., Moraes, T. B., Linder, C., \& Wiesman, Z. (2015) Liquid phase characterization of molecular interactions in polyunsaturated and $\mathrm{n}$-fatty acid methyl esters by $1 \mathrm{H}$ low field nuclear magnetic resonance. Biotechnology for Biofuels ,8:96-108.

Merkx, D. W. H., Hong, G. T. S., Ermacora, A., \& van Duynhoven, J. P. M. (2018) Rapid Quantitative Profiling of Lipid Oxidation Products in a Food Emulsion by 1H NMR. Analytical Chemistry , 90 : 48634870.

Millichip, M., Tatham, A. S., Jackson, F., Griffiths, G., Shewry, P. R., \& Stobart, A. K. (1996). Purification and characterization of oil-bodies (oleosomes) and oil-body boundary proteins (oleosins) from the developing cotyledons of sunflower (Helianthus annuus L.). The Biochemical journal , 314 (Pt 1), 333-337. https://doi.org/10.1042/bj3140333

Miyashita, K., Nara, E., \& Ota, T. (1993) Oxidative Stability of Polyunsaturated Fatty Acids in an Aqueous Solution. Bioscience, Biotechnology, and Biochemistry, 57 : 1638-1640.

Miyashita, K., Uemura, M., \& Hosokawa, M. (2018) Effective Prevention of Oxidative Deterioration of Fish Oil: Focus on Flavor Deterioration. Annual Review of Food Science and Technology, 9 : 209-226.

Nikiforidis, C. V. (2019) Structure and functions of oleosomes (oil bodies).Advances in Colloid and Interface Science, 274 : 102039-102044.

Nikovska, K. (2012) Study of olive oil-in-water emulsions with protein emulsifiers. Emirates Journal of Food and Agriculture, 24 :17-24.

Purkrtova, Z., Jolivet, P., Miquel, M., \& Chardot, T. (2008) Structure and Function of Seed Lipid BodyAssociated Proteins. Comptes Rendus Biologies, 331 : 746- 754.

Resende, M. T., Campisi-Pinto, S., Linder, C., \& Wiesman, Z. (2019a) Multidimensional Proton Nuclear Magnetic Resonance Relaxation Morphological and Chemical Spectrum Graphics for Monitoring and Characterization of Polyunsaturated Fatty-Acid Oxidation. Journal of the American Oil Chemists' Society, 96 :125-135.

Resende, M. T., Linder, C., \& Wiesman, Z. (2019b) ${ }^{1}$ H LF-NMR Energy Relaxation Time Characterization of the Chemical and Morphological Structure of PUFA-Rich Linseed Oil During Oxidation With and Without Antioxidants. European Journal of Lipid Science and Technology , 121 : 1800339-1800347.

Resende, M. T., Linder, C., \& Wiesman, Z. (2020) Alkyl Tail Segments Mobility as a Marker for Omega-3 PUFA-rich Linseed Oil Oxidative Aging. Journal of the American Oil Chemists' Society. (in press).

Shen, Z., Wijesundera, C., \& Ye, J.-H. (2012) Effect of Seed Heat-Treatment on the Oxidative Stability of Canola Oil Body Emulsions.Food and Nutrition Sciences, 3 : 981-990.

Song, Y., Venkataramanan, L., Hurlimann, M. D., Flaum, M., Frulla, P., \& Straley, C. (2002) T1-T2 Correlation Spectra Obtained Using a Fast Two-Dimensional Laplace Inversion. Journal of Magnetic Resonance ,154:261-268.

Stejskal, E. O., \& Tanner, J. E. (1965) Spin Diffusion Measurements: Spin Echoes in the Presence of a Time-Dependent Field Gradient. The Journal of Chemical Physics , 42 :288-292.

Sun, Y-E., Wang, W-D., Chen, H-W., \& Li, C. (2011) Autoxidation of unsaturated lipids in food emulsion. Journal Critical Reviews in Food Science and Nutrition, 51 :453-466. 
Takur, D., Bora, T. C., Mazumbar, S., Bordolol, G. N. (2009) Influence of nutrition and culturing conditions for optimum growth and antimicrobial metabolite production by Streptomyces sp. 201.Journal of Medical Mycology, 19 :161-167.

Thompson, L. U., \& Cunnane, S. C. (2003) Flaxseed in human nutrition. Champaign, IL: AOCS Press.

Tzen, T. C., \& Huang, A. H. C. (1992) Surface Structure and Properties of Plant Seed Oil Bodies. Cell Biology, 117 : 327-335.

Zarate, R., El Jaber-Vazdekis, N., Tejera, N., Perez, J. A., \& Rodriguez, C. (2017) Significance of long chain polyunsaturated fatty acids in human health. Clinical and Translational Medicine ,6: 25.

Velasco, J., Andersen, M. L., \& Skibsted, L. H. (2005) Electron Spin Resonance Spin Trapping for Analysis of Lipid Oxidation in Oils: Inhibiting Effect of the Spin Trap r-Phenyl-N-tert-butylnitrone on Lipid Oxidation. Journal of Agricultural and Food Chemistry, 53 :1328-1336.

Wahlroos, T., Soukka, J., Denesyuk, A. \& Susi, P. (2015) Amino-Terminus of Oleosin Protein Defines the Size of Oil Bodies - Topological Model of Oleosin-Oil Body Complex. Journal of Plant Biochemistry \& Physiology, 3 : 1000155-1000160.

Wiesman, Z., Linder, C., Resende, M. T., Ayalon, N., Levi, O., Bernardinelli, O. D., Jackman, R. (2018) 2D and 3D Spectrum graphics of the chemical-morphological domains of complex biomass by low field proton NMR energy relaxation signal analysis. Energy \& Fuels, 32 :5090-5102.

Yehuda, S., Rabinovitz, S., Mostofsky, D. I. (1999) Essential fattyj acids are mediators of brain biochemistry and cognitive functions. Journal of Neuroscience Research , 56 : 565-570.

Yesiltas, B., Garcia-Moreno, P. J., Sorensen, A-D. M., Akoh, C. C., \& Jacobsen, C. (2019). Physical and oxidative stability of high fat fish oil-in-water emulsions stabilized with sodium caseinate and phosphatidylcholine as emulsifiers. Food Chemistry, 276 : 110-118 